\title{
INEQUALITIES FOR POISSON INTEGRALS WITH SLOWLY GROWING DIMENSIONAL CONSTANTS
}

\author{
Loukas Grafakos*, Enrico LaenG**, and Carlo Morpurgo***
}

Abstract

Let $P_{t}$ be the Poisson kernel. We study the following $L^{p}$ inequality for the Poisson integral $P f(x, t)=\left(P_{t} * f\right)(x)$ with respect to a Carleson measure $\mu$ :

$$
\|P f\|_{L^{p}\left(\mathbb{R}_{+}^{n+1}, d \mu\right)} \leq c_{p, n} \kappa(\mu)^{\frac{1}{p}}\|f\|_{L^{p}\left(\mathbb{R}^{n}, d x\right)},
$$

where $1<p<\infty$ and $\kappa(\mu)$ is the Carleson norm of $\mu$. It was shown by Verbitsky $[\mathbf{V}]$ that for $p>2$ the constant $c_{p, n}$ can be taken to be independent of the dimension $n$. We show that $c_{2, n}=O\left((\log n)^{\frac{1}{2}}\right)$ and that $c_{p, n}=O\left(n^{\frac{1}{p}-\frac{1}{2}}\right)$ for $1<p<2$ as $n \rightarrow \infty$. We observe that standard proofs of this inequality rely on doubling properties of cubes and lead to a value of $c_{p, n}$ that grows exponentially with $n$.

\section{Introduction}

The object of study in this article is the following Carleson measure inequality $[\mathbf{C 1}],[\mathbf{C 2}]$, valid for $1<p<\infty$

$$
\|P f\|_{L^{p}\left(\mathbb{R}_{+}^{n+1}, d \mu\right)} \leq c_{p, n} \kappa(\mu)^{\frac{1}{p}}\|f\|_{L^{p}\left(\mathbb{R}^{n}\right)},
$$

where $\mu$ is a Carleson measure on $\mathbb{R}_{+}^{n+1}$ with norm $\kappa(\mu)$ and $P$ is the Poisson integral of a function $f$ on $\mathbb{R}^{n}$.

We begin by recalling these notions and establishing notation. We denote by $\bar{B}\left(x_{0}, r\right)$ the closed ball in $\mathbb{R}^{n}$ with radius $r$ centered at $x_{0}$. The Carleson tent $T\left[\bar{B}\left(x_{0}, r\right)\right]$ over the ball $\bar{B}\left(x_{0}, r\right)$ is defined as the

2000 Mathematics Subject Classification. Primary: 42B25; Secondary: 31B05. Key words. Carleson measures, harmonic functions, dimension free estimates. * Supported in part by the NSF Grant DMS-0400387.

**Supported in part by the Italian Fondi Ministeriali MIUR.

***Supported by the NSF Grant DMS-0200574. 
set of all points $(x, t) \in \mathbb{R}^{n} \times \mathbb{R}^{+}$such that $\left|x-x_{0}\right|^{2}+t^{2}<r^{2}$. Denote by $|K|$ the Lebesgue measure of a set $K$ in $\mathbb{R}^{n}$. A Borel measure $\mu$ on $\mathbb{R}_{+}^{n+1}$ is called a Carleson measure if its Carleson norm $\kappa(\mu)=$ $\sup \left\{\frac{\mu\left(T\left[\bar{B}\left(x_{0}, r\right)\right]\right)}{\left|\bar{B}\left(x_{0}, r\right)\right|}: x_{0} \in \mathbb{R}^{n}, r>0\right\}$ is a finite number.

We define the Poisson integral $P f$, i.e., the harmonic extension of $f$ to $\mathbb{R}_{+}^{n+1}$, as the convolution

$$
P f(x, a)=P_{a} * f(x)=\int_{\mathbb{R}^{n}} P_{a}(x-y) f(y) d y,
$$

where the Poisson kernel is defined, for all $a>0, x \in \mathbb{R}^{n}$ by

$$
P_{a}(x)=\frac{\gamma_{n} a}{\left(a^{2}+|x|^{2}\right)^{\frac{n+1}{2}}}, \quad \text { with } \quad \gamma_{n}=\frac{\Gamma\left(\frac{n+1}{2}\right)}{\pi^{\frac{n+1}{2}}} .
$$

The Lebesgue measure of the closed unit ball $\bar{B}(0,1)$ and the surface measure of its boundary are denoted by

(1.4) $\Omega_{n}=|\bar{B}(0,1)|=\frac{\pi^{\frac{n}{2}}}{\Gamma\left(\frac{n}{2}+1\right)}, \quad \omega_{n-1}=|\partial \bar{B}(0,1)|=\frac{2 \pi^{\frac{n}{2}}}{\Gamma\left(\frac{n}{2}\right)}=n \Omega_{n}$.

Verbitsky [V] gave an elegant proof of (1.1) that yields a constant $c_{p, n}$ independent of $n$ whenever $p>2$. The starting point of Verbitsky's argument is the use of interpolation and duality to derive (1.1) from the equivalent inequalities

$$
\begin{aligned}
& \|P f\|_{L^{2, \infty}\left(\mathbb{R}_{+}^{n+1}, d \mu\right)} \leq c^{\prime} \kappa(\mu)^{\frac{1}{2}}\|f\|_{L^{2}\left(\mathbb{R}^{n}\right)}, \\
& \left\|P^{*}(g, \mu)\right\|_{L^{2}\left(\mathbb{R}^{n}\right)} \leq c^{\prime} \kappa(\mu)^{\frac{1}{2}}\|g\|_{L^{2,1}\left(\mathbb{R}_{+}^{n+1}, d \mu\right)},
\end{aligned}
$$

for some absolute constant $c^{\prime}$ independent of $n$, where $P^{*}$ is the Balayage operator defined for functions $g$ on $\mathbb{R}_{+}^{n+1}$ by

$$
P^{*}(g, \mu)(x)=\int_{\mathbb{R}_{+}^{n+1}} P_{b}(x-y) g(y, b) d \mu(y, b) .
$$

Using the semigroup property for the Poisson kernel,

$$
\int_{\mathbb{R}^{n}} P_{a}(\tau-x) P_{b}(\tau-y) d \tau=P_{a+b}(x-y),
$$

Verbitsky obtained (1.6) with $c^{\prime}$ independent of $n$ for characteristic functions of subsets of $\mathbb{R}_{+}^{n+1}$ and this is enough to establish (1.6) for general $\mu$-measurable functions $g$ (see $[\mathbf{S W}]$ ).

The following theorem is the main result of this article, which grew out of our attempts to extend Verbitsky's theorem to the case $1<p \leq 2$ : 
Theorem 1.1. For $1<p<2$ the following Carleson measure inequality holds:

$$
\|P f\|_{L^{p}\left(\mathbb{R}_{+}^{n+1}, d \mu\right)} \leq C_{p} n^{\frac{1}{p}-\frac{1}{2}} \kappa(\mu)^{\frac{1}{p}}\|f\|_{L^{p}\left(\mathbb{R}^{n}\right)},
$$

with $C_{p}$ independent of $n$. For $p=2$ the following holds:

$$
\|P f\|_{L^{2}\left(\mathbb{R}_{+}^{n+1}, d \mu\right)} \leq C(\log n)^{\frac{1}{2}} \kappa(\mu)^{\frac{1}{2}}\|f\|_{L^{2}\left(\mathbb{R}^{n}\right)},
$$

with $C$ independent of $n$.

We observe that the estimate above is sharper than those obtained from the other known proofs of the Carleson measure inequality (1.1) (see, e.g., $[\mathbf{A}],[\mathbf{C 2}],[\mathbf{G}],[\mathbf{H}],[\mathbf{N}],[\mathbf{S}]$ ); these proofs yield constants that grow exponentially in $n$.

The authors would like to thank Igor Verbitsky for pointing out this problem to them and for sharing some of his ideas with them.

\section{An integral formula involving Poisson kernels}

In order to extend the arguments in $[\mathbf{V}]$ we are lead to consider the equivalent inequalities for $k=3,4,5, \ldots$

$$
\begin{aligned}
& \|P f\|_{L^{\frac{k}{k-1}, \infty}\left(\mathbb{R}_{+}^{n+1}, d \mu\right)} \leq \bar{c}(k, n) \kappa(\mu)^{\frac{k-1}{k}}\|f\|_{L^{\frac{k}{k-1}}\left(\mathbb{R}^{n}\right)}, \\
& \left\|P^{*}(g, \mu)\right\|_{L^{k}\left(\mathbb{R}^{n}\right)} \leq \bar{c}(k, n) \kappa(\mu)^{\frac{k-1}{k}}\|g\|_{L^{k, 1}\left(\mathbb{R}_{+}^{n+1}, d \mu\right)}, \\
& \left\|P^{*}\left(\chi_{E}, \mu\right)\right\|_{L^{k}\left(\mathbb{R}^{n}\right)} \leq c(k, n) \kappa(\mu)^{\frac{k-1}{k}} \mu(E)^{\frac{1}{k}}, \quad \forall E \subset \mathbb{R}_{+}^{n+1} .
\end{aligned}
$$

For $k=2$ these inequalities hold with $c(2, n) \leq c_{2, n} \leq c_{2}$ independent of $n$, due to $[\mathbf{V}]$, (see (1.5) above). Our goal is to obtain good estimates for the constants $c(k, n)$ for all $k=3,4, \ldots$ and then use the Marcinkiewicz interpolation theorem to deduce (1.1).

Since

$\left\|P^{*}\left(\chi_{E}, \mu\right)\right\|_{L^{k}\left(\mathbb{R}^{n}\right)}^{k}=\int_{E^{k}} \int_{\mathbb{R}^{n}} \prod_{j=1}^{k} P_{a_{j}}\left(x_{j}-\tau\right) d \tau d \mu\left(x_{1}, a_{1}\right) \ldots d \mu\left(x_{k}, a_{k}\right)$

our first task is to derive a workable formula for

$$
\int_{\mathbb{R}^{n}} \prod_{j=1}^{k} P_{a_{j}}\left(x_{j}-\tau\right) d \tau
$$

which, in the case $k=2$, is computed explicitly via the semigroup property. In this section we prove the following: 
Proposition 2.1. Let $P_{a}(x)$ be the Poisson kernel, as in definition (1.3). Then, for any $a_{1}, \ldots, a_{k} \in(0, \infty)$ and for any $x_{1}, \ldots, x_{k} \in \mathbb{R}^{n}$, we have

$$
\begin{aligned}
\int_{\mathbb{R}^{n}} \prod_{j=1}^{k} P_{a_{j}}\left(\tau-x_{j}\right) d \tau & \\
= & \frac{a_{1} a_{2} \ldots a_{k}}{\pi^{(k-1) \frac{n}{2}+\frac{k}{2}}} \int_{0}^{\infty} \cdots \int_{0}^{\infty} \frac{\left(u_{1} u_{2} \ldots u_{k}\right)^{\frac{n-1}{2}}}{\left(u_{1}+u_{2}+\cdots+u_{k}\right)^{\frac{n}{2}}} \\
& \times \operatorname{Exp}\left[-\sum_{j=1}^{k} a_{j}^{2} u_{j}-\frac{\sum_{1 \leq i<j \leq k} u_{i} u_{j}\left|x_{i}-x_{j}\right|^{2}}{u_{1}+u_{2}+\cdots+u_{k}}\right] d u_{1} \ldots d u_{k} .
\end{aligned}
$$

In particular, when $k=3$, the formula above can be written as

$$
\begin{aligned}
\int_{\mathbb{R}^{n}} P_{a}(\tau-x) P_{b}(\tau-y) P_{c}(\tau-z) d \tau & \\
= & \frac{a b c}{\pi^{n+\frac{3}{2}}} \int_{0}^{\infty} \int_{0}^{\infty} \int_{0}^{\infty} \frac{(u v w)^{\frac{n-1}{2}}}{(u+v+w)^{\frac{n}{2}}} \\
& \times \operatorname{Exp}\left[-\left\{a^{2} u+b^{2} v+c^{2} w\right.\right. \\
& \left.\left.+\frac{u v|x-y|^{2}+u w|x-z|^{2}+v w|y-z|^{2}}{u+v+w}\right\}\right] d u d v d w
\end{aligned}
$$

and when $k=2$, we have

$$
\begin{aligned}
\int_{\mathbb{R}^{n}} & P_{a}(\tau-x) P_{b}(\tau-y) d \tau \\
& =\frac{a b}{\pi^{\frac{n}{2}+1}} \int_{0}^{+\infty} \int_{0}^{+\infty} \frac{(u v)^{\frac{n-1}{2}}}{(u+v)^{\frac{n}{2}}} e^{-\left\{a^{2} u+b^{2} v+\frac{u v}{u+v}|x-y|^{2}\right\}} d u d v
\end{aligned}
$$

Proof: We start from the "subordination" formula

$$
P_{a}(x)=\int_{0}^{+\infty} \beta_{a}(u) e^{-u|x|^{2}} d u
$$

where

$$
\beta_{a}(u)=\frac{a}{\pi^{\frac{n+1}{2}}} e^{-a^{2} u} u^{\frac{n-1}{2}}
$$

which can be easily deduced using the definition of the Gamma function. 
The integral on the left hand side in (2.1) can now be rewritten as

$$
\begin{aligned}
\int_{\mathbb{R}^{n}} & \prod_{j=1}^{k} P_{a_{j}}\left(\tau-x_{j}\right) d \tau \\
& =\int_{\mathbb{R}^{n}} \prod_{j=1}^{k}\left(\int_{0}^{+\infty} \beta_{a_{j}}\left(u_{j}\right) e^{-u_{j}\left|\tau-x_{j}\right|^{2}} d u_{k}\right) d \tau \\
= & \frac{a_{1} \ldots a_{k}}{\pi^{\frac{(n+1) k}{2}}} \int_{\mathbb{R}^{n}} \int_{[0, \infty)^{k}}\left(u_{1} \ldots u_{k}\right)^{\frac{n-1}{2}} e^{-\sum_{j=1}^{k}\left(a_{j}^{2} u_{j}+u_{j}\left|\tau-x_{j}\right|^{2}\right)} d u_{1} \ldots d u_{k} d \tau \\
= & \frac{a_{1} \ldots a_{k}}{\pi^{\frac{(n+1) k}{2}}} \int_{[0, \infty)^{k}}\left(u_{1} \ldots u_{k}\right)^{\frac{n-1}{2}} e^{-\sum_{j=1}^{k} a_{j}^{2} u_{j}} \\
\times & \times\left[\int_{\mathbb{R}^{n}} e^{-\sum_{j=1}^{k} u_{j}\left|\tau-x_{j}\right|^{2}} d \tau\right] d u_{1} \ldots d u_{k}
\end{aligned}
$$

Let us now rewrite the inner integral inside square brackets using Cartesian coordinates, namely $\tau=\left(\tau_{1}, \ldots, \tau_{n}\right)$ and $x_{j}=\left(x_{j 1}, \ldots, x_{j n}\right)$. We obtain

$$
\begin{aligned}
& \int_{\mathbb{R}^{n}} e^{-\sum_{j=1}^{k} u_{j}\left|\tau-x_{j}\right|^{2}} d \tau \int_{\mathbb{R}^{n}} e^{-\left\{u_{1}\left(\tau_{1}-x_{11}\right)^{2}+u_{2}\left(\tau_{1}-x_{21}\right)^{2}+\cdots+u_{k}\left(\tau_{1}-x_{k 1}\right)^{2}\right\}} \ldots \\
& \ldots e^{-\left\{u_{1}\left(\tau_{n}-x_{1 n}\right)^{2}+u_{2}\left(\tau_{n}-x_{2 n}\right)^{2}+\cdots+u_{k}\left(\tau_{n}-x_{k n}\right)^{2}\right\}} d \tau_{1} d \tau_{2} \ldots d \tau_{n}=\prod_{r=1}^{n} I_{r}
\end{aligned}
$$

where, for each index $r=1,2, \ldots, n$, we have defined

$$
I_{r}=\int_{-\infty}^{+\infty} e^{-\left(A \tau_{r}^{2}-2 B \tau_{r}+C\right)} d \tau_{r}
$$

with

$$
\begin{aligned}
& A=u_{1}+u_{2}+\cdots+u_{k} \\
& B=B_{r}=u_{1} x_{1 r}+u_{2} x_{2 r}+\cdots+u_{k} x_{k r} \\
& C=C_{r}=u_{1} x_{1 r}^{2}+u_{2} x_{2 r}^{2}+\cdots+u_{k} x_{k r}^{2} .
\end{aligned}
$$


We claim that, for each choice of the coordinate index $r=1, \ldots, n$ we have

$$
B^{2}-A C=B_{r}^{2}-A C_{r}=-\sum_{i<j} u_{i} u_{j}\left(x_{i r}-x_{j r}\right)^{2}
$$

where the sum is taken over all possible pairs of distinct indices $i$ and $j$ both running from 1 to $k$ (there are $k(k-1) / 2$ of such pairs). In fact this claim is easily checked by observing that in $B^{2}-A C$ all the square terms of the kind $u_{i}^{2} x_{i r}^{2}$ cancel out, while the remaing mixed terms can be collected in groups of three, each group giving $-u_{i} u_{j}\left(x_{i r}^{2}-2 x_{i r} x_{j r}+x_{j r}^{2}\right)$.

Now, completing the square in $(2.5)$ we obtain

$I_{r}=e^{\frac{B^{2}-A C}{A}} \int_{-\infty}^{+\infty} e^{-A\left(\tau_{r}-\frac{B}{A}\right)^{2}} d \tau_{r}=\sqrt{\frac{\pi}{u_{1}+\cdots+u_{k}}} e^{-\frac{\sum_{i<j} u_{i} u_{j}\left(x_{i r}-x_{j r}\right)^{2}}{u_{1}+\cdots+u_{k}}}$

which implies that

$$
\int_{\mathbb{R}^{n}} e^{-\sum_{j=1}^{k} u_{j}\left|\tau-x_{j}\right|^{2}} d \tau=\left(\frac{\pi}{u_{1}+\cdots+u_{k}}\right)^{\frac{n}{2}} e^{-\frac{\sum_{i<j} u_{i} u_{j}\left|x_{i}-x_{j}\right|^{2}}{u_{1}+\cdots+u_{k}}}
$$

Using this identity in the square brackets in (2.4) and simplifying, we obtain (2.1).

Remark. The reader may wonder if it is possible to obtain a formula for the left hand side in (2.1) that does not involve any integrals, something analogous to (1.7) for $k \geq 3$. When $n=1$ using residues (plus some involved algebraic manipulations) we were able to show that

$$
\begin{aligned}
& \int_{\mathbb{R}} P_{a}(\tau-x) P_{b}(\tau-y) P_{c}(\tau-z) d \tau \\
&= \frac{a b}{(a+c)(b+c)} P_{a+c}(x-z) P_{b+c}(y-z) \\
&+\frac{a c}{(a+b)(c+b)} P_{b+c}(y-z) P_{a+b}(x-y) \\
&+\frac{b c}{(b+a)(c+a)} P_{a+c}(x-z) P_{a+b}(x-y) \\
&+4 \pi \frac{a b c(a+b+c)}{(a+b)(a+c)(b+c)} P_{a+b}(x-y) P_{a+c}(x-z) P_{b+c}(y-z)
\end{aligned}
$$


Unfortunately, for $n>1$, an integral-free formula of this sort is difficult to obtain. It seems that the size and complexity of the formula grows quickly with $n$, and furthermore, there is no obvious "leading term" to be used in our estimates as $n \rightarrow \infty$. On the other hand we will show that (2.1), after some manipulations, suffices for the purposes of the proof of Theorem 1.1.

\section{Proof of Theorem 1.1: the case $\frac{3}{2}<p<2$}

For clarity of exposition we first give a detailed proof of Theorem 1.1 in the case $3 / 2<p<2$. In the next section we indicate how the same technique can be adapted to the case $p \in\left(\frac{k}{k-1}, 2\right)$, any $k=4,5, \ldots$.

Let us start by showing that the Balayage operator $P^{*}$ satisfies the following estimate for all Carleson measures $\mu$, for all $\mu$-measurable subsets $E$ of $\mathbb{R}_{+}^{n+1}$, and for all $n=1,2, \ldots$,

$$
\left\|P^{*}\left(\chi_{E}, \mu\right)\right\|_{L^{3}\left(\mathbb{R}^{n}\right)}^{3} \leq c^{\prime \prime} n^{\frac{1}{2}} \kappa(\mu)^{2} \mu(E),
$$

for some absolute constant $c^{\prime \prime}$. Once (3.1) is established, using duality and $[\mathbf{S W}]$ we obtain for some other absolute constant $c^{\prime \prime \prime}$

$$
\|P f\|_{L^{\frac{3}{2}, \infty}\left(\mathbb{R}_{+}^{n+1}, d \mu\right)} \leq c^{\prime \prime \prime} n^{\frac{1}{6}} \kappa(\mu)^{\frac{2}{3}}\|f\|_{L^{\frac{3}{2}}\left(\mathbb{R}^{n}\right)}
$$

and thus, by the Marcinkiewicz interpolation and (1.5), Theorem 1.1 follows in the case $3 / 2<p<2$.

We have

$$
\begin{aligned}
& \quad\left\|P^{*}\left(\chi_{E}, \mu\right)\right\|_{L^{3}\left(\mathbb{R}^{n}\right)}^{3} \\
& =\int_{E^{3}} \int_{\mathbb{R}^{n}} P_{a}(\tau-x) P_{b}(\tau-y) P_{c}(\tau-z) d \tau d \mu(x, a) d \mu(y, b) d \mu(z, c) .
\end{aligned}
$$

We will use the following modification of formula (2.2):

Lemma 3.1. The integral in (2.2) can also be written as

$$
\begin{aligned}
& \int_{\mathbb{R}^{n}} P_{a}(\tau-x) P_{b}(\tau-y) P_{c}(\tau-z) d \tau \\
& =\frac{\Gamma\left(\frac{n+3}{2}\right)}{\pi^{n+\frac{3}{2}}} a b c \int_{0}^{1} \int_{0}^{1} \frac{(1-t)^{n} t^{\frac{n-1}{2}}(s(1-s))^{\frac{n-1}{2}}}{\left\{a^{2} t+(1-t) B^{2}+t(1-t)|x-q|^{2}\right\}^{n+\frac{3}{2}}} d t d s
\end{aligned}
$$


where

$$
\begin{gathered}
B^{2}=B^{2}(s, y, z)=b^{2} s+c^{2}(1-s)+s(1-s)|y-z|^{2} \\
q=q(s, y, z)=s y+(1-s) z
\end{gathered}
$$

We will also need the following estimates:

Lemma 3.2. The following inequalities hold for $\alpha>0, \beta>0, \gamma>0$ such that $\beta>\alpha$ and $\gamma<\beta+2$ and for any $D \in \mathbb{R}$.

$$
\begin{aligned}
\int_{0}^{1} t^{\alpha}(1-t)^{\beta} & \left\{a^{2} t+(1-t) B^{2}+t(1-t) D^{2}\right\}^{-\gamma} d t \\
& \leq \frac{1}{a B} \int_{0}^{1} t^{\alpha}(1-t)^{\beta}\left\{a^{2} t+(1-t) B^{2}+t(1-t) D^{2}\right\}^{-\gamma+1} d t \\
& \leq \frac{1}{a B} \int_{0}^{1} t^{\alpha}(1-t)^{\beta-\gamma+1}\left\{B^{2}+t\left(a^{2}+D^{2}\right)\right\}^{-\gamma+1} d t
\end{aligned}
$$

The proofs of these lemmas are postponed until the end of this section.

Returning to the proof of (3.1), we split (3.3) in the six regions $a \leq$ $b \leq c, a \leq c \leq b, b \leq c \leq a, b \leq a \leq c, c \leq b \leq a$, and $c \leq a \leq b$; by symmetry we need only consider the region over which $a \leq b \leq c$. Following $[\mathbf{V}]$ we write

$$
\begin{aligned}
&\left\|P^{*}\left(\chi_{E}, \mu\right)\right\|_{L^{3}\left(\mathbb{R}^{n}\right)}^{3}(6 \mu(E))^{-1} \\
& \leq \sup _{(z, c) \in E} \int_{\mathbb{R}^{n} \times(0, c]}\left[\int_{\mathbb{R}^{n} \times(0, b]} \int_{\mathbb{R}^{n}} P_{a}(\tau-x)\right. \\
&\left.\times P_{b}(\tau-y) P_{c}(\tau-z) d \tau d \mu(x, a)\right] d \mu(y, b) .
\end{aligned}
$$

Observe that $a \leq b \leq c$ implies $a \leq B$. Applying Lemma 3.2 for each fixed $s,(y, b)$, and $(z, c)$, with $B$ and $q$ as in (3.4) and (3.5), $D=|x-q|$, 
$\alpha=(n-1) / 2, \beta=n$ and $\gamma=n+3 / 2$, we have

$$
\begin{aligned}
& \int_{\mathbb{R}^{n} \times(0, b]} \frac{\Gamma\left(n+\frac{3}{2}\right)}{\pi^{n+\frac{3}{2}}} a b c \int_{0}^{1} \frac{t^{\frac{n-1}{2}}(1-t)^{n}}{\left(a^{2} t+(1-t) B^{2}+t(1-t)|x-q|^{2}\right)^{n+\frac{3}{2}}} d t d \mu(x, a) \\
& \leq \int_{\mathbb{R}^{n} \times(0, b]} \frac{b c \Gamma\left(n+\frac{3}{2}\right)}{B \pi^{n+\frac{3}{2}}} \int_{0}^{1} \frac{t^{\frac{n-1}{2}}(1-t)^{-1 / 2}}{\left(B^{2}+t\left(a^{2}+|x-q|^{2}\right)\right)^{n+\frac{1}{2}}} d t d \mu(x, a) \\
& \leq \frac{b c\left(n+\frac{1}{2}\right) \Gamma\left(n+\frac{3}{2}\right)}{B \pi^{n+\frac{3}{2}}} \int_{0}^{1} \int_{\mathbb{R}_{+}^{n+1}} \int_{B^{2}+t\left(a^{2}+|x-q|^{2}\right)}^{\infty} \frac{t^{\frac{n-1}{2}}(1-t)^{-\frac{1}{2}}}{r^{n+\frac{3}{2}}} d t d r d \mu(x, a) \\
& \leq \frac{b c\left(n+\frac{1}{2}\right) \Gamma\left(n+\frac{3}{2}\right)}{B \pi^{n+\frac{3}{2}}} \int_{0}^{1} \int_{B^{2}}^{\infty} \mu\left(T\left[\bar{B}\left(q,\left(\frac{r-B^{2}}{t}\right)^{\frac{1}{2}}\right)\right]\right) \\
& \times \frac{t^{\frac{n-1}{2}}(1-t)^{-\frac{1}{2}}}{r^{n+\frac{3}{2}}} d t d r d \mu(x, a) \\
& \leq \Omega_{n} \kappa(\mu) \frac{b c\left(n+\frac{1}{2}\right) \Gamma\left(n+\frac{3}{2}\right)}{B \pi^{n+\frac{3}{2}}} \int_{0}^{1} \int_{B^{2}}^{\infty}\left(r-B^{2}\right)^{\frac{n}{2}} \\
& \times \frac{t^{-\frac{1}{2}}(1-t)^{-\frac{1}{2}}}{r^{n+\frac{3}{2}}} d t d r d \mu(x, a) \\
& =\kappa(\mu) \frac{b c\left(n+\frac{1}{2}\right) \Gamma\left(n+\frac{3}{2}\right)}{B^{n+2} \pi^{\frac{n+3}{2}} \Gamma\left(\frac{n}{2}+1\right)} \int_{0}^{1} \int_{1}^{\infty} \frac{(r-1)^{\frac{n}{2}}}{r^{n+\frac{3}{2}}} t^{-\frac{1}{2}}(1-t)^{-\frac{1}{2}} d t d r d \mu(x, a) \\
& =\kappa(\mu) \frac{b c\left(n+\frac{1}{2}\right) \Gamma\left(\frac{n+1}{2}\right)}{B^{n+2} \pi^{\frac{n+1}{2}}} \\
& =\kappa(\mu) \frac{b c\left(n+\frac{1}{2}\right) \Gamma\left(\frac{n+1}{2}\right)}{\pi^{\frac{n+1}{2}}\left(b^{2} s+c^{2}(1-s)+s(1-s)|y-z|^{2}\right)^{\frac{n}{2}+1}} .
\end{aligned}
$$

Next, observe that the following identity holds:

$$
\begin{aligned}
& \int_{0}^{1} \frac{b c(s(1-s))^{\frac{n-1}{2}}}{\left(b^{2} s+c^{2}(1-s)+s(1-s)|y-z|^{2}\right)^{\frac{n}{2}+1}} d s \\
& \quad=\frac{\gamma_{n} \pi^{\frac{n}{2}+1}}{\Gamma\left(\frac{n}{2}+1\right)} \frac{(b+c)}{\left[(b+c)^{2}+|y-z|^{2}\right]^{\frac{n+1}{2}}} .
\end{aligned}
$$


In fact, by the semigroup formula for the Poisson kernel and (2.3) we have

$$
P_{b+c}(y-z)=\frac{b c}{\pi^{\frac{n}{2}+1}} \int_{0}^{+\infty} \int_{0}^{+\infty} \frac{(u v)^{\frac{n-1}{2}}}{(u+v)^{\frac{n}{2}}} e^{-\left(b^{2} u+c^{2} v+\frac{u v}{u+v}|y-z|^{2}\right)} d u d v
$$

On the other hand, after the change of variables $v=\lambda u$ the above quantity becomes

$$
\begin{aligned}
\frac{b c}{\pi^{\frac{n}{2}+1}} \int_{0}^{+\infty} \frac{\lambda^{\frac{n-1}{2}}}{(1+\lambda)^{\frac{n}{2}}} d \lambda \int_{0}^{+\infty} u^{\frac{n}{2}} e^{-W u} d u & \\
& =\frac{b c \Gamma\left(\frac{n}{2}+1\right)}{\pi^{\frac{n}{2}+1}} \int_{0}^{+\infty} \frac{\lambda^{\frac{n-1}{2}}}{(1+\lambda)^{\frac{n}{2}} W^{\frac{n}{2}+1}} d \lambda
\end{aligned}
$$

where $W=b^{2}+c^{2} \lambda+\frac{\lambda}{1+\lambda}|y-z|^{2}$, and the further change of variables $s=1 /(\lambda+1)$ (and therefore $\lambda=(1-s) / s$ ) gives

$P_{b+c}(y-z)=\frac{\Gamma\left(\frac{n}{2}+1\right)}{\pi^{\frac{n}{2}+1}} \int_{0}^{1} \frac{b c(s(1-s))^{\frac{n-1}{2}}}{\left(b^{2} s+c^{2}(1-s)+s(1-s)|y-z|^{2}\right)^{\frac{n}{2}+1}} d s$.

We now apply Lemma 3.1 in the inner most integral in (3.6) and then use estimate $(3.7)$ integrated with respect to $(s(1-s))^{\frac{n-1}{2}} d s d \mu(y, b)$ on $[0,1] \times \mathbb{R}^{n} \times(0, c]$ and identity (3.8) to obtain

$$
\begin{gathered}
\int_{\mathbb{R}^{n} \times(0, c]}\left[\int_{\mathbb{R}^{n} \times(0, b]} \int_{\mathbb{R}^{n}} P_{a}(\tau-x) P_{b}(\tau-y) P_{c}(\tau-z) d \tau d \mu(x, a)\right] d \mu(y, b) \\
\leq \kappa(\mu) \frac{\left(n+\frac{1}{2}\right) \Gamma\left(\frac{n+1}{2}\right)}{\pi^{\frac{n+1}{2}}} \int_{\mathbb{R}_{+}^{n+1}} \frac{\gamma_{n} \pi^{\frac{n}{2}+1}}{\Gamma\left(\frac{n}{2}+1\right)} \frac{(b+c)}{\left[(b+c)^{2}+|y-z|^{2}\right]^{\frac{n+1}{2}}} d \mu(y, b) \\
\leq \kappa(\mu) \frac{2 \pi^{1 / 2}\left(n+\frac{1}{2}\right) \Gamma\left(\frac{n+1}{2}\right)}{\Gamma\left(\frac{n}{2}+1\right)} \int_{\mathbb{R}_{+}^{n+1}} \gamma_{n} \frac{c}{\left[(b+c)^{2}+|y-z|^{2}\right]^{\frac{n+1}{2}}} d \mu(y, b) .
\end{gathered}
$$

By (13) in $[\mathbf{V}]$

$$
\sup _{(z, c) \in \mathbb{R}_{+}^{n+1}} \int_{\mathbb{R}_{+}^{n+1}} \gamma_{n} \frac{c}{\left[(b+c)^{2}+|y-z|^{2}\right]^{\frac{n+1}{2}}} d \mu(y, b) \leq C \kappa(\mu)
$$

for some absolute constant $C$ independent of $n$. Therefore (3.9) is bounded above by some absolute constant independent of $n$ times

$$
\kappa(\mu)^{2} \frac{\left(n+\frac{1}{2}\right) \Gamma\left(\frac{n+1}{2}\right)}{\Gamma\left(\frac{n}{2}+1\right)} \sim \kappa(\mu)^{2} \sqrt{n}
$$


as $n \rightarrow \infty$, by the well known expansion $\Gamma(z) / \Gamma(z+\alpha) \sim z^{-\alpha}$ for $z \rightarrow \infty$. This last bound for (3.9), together with (3.6), proves (3.1) and hence Theorem 1.1 in the case $3 / 2<p<2$.

Proof of Lemma 3.1: We start with the following $n$-dimensional identity (3.10) $\frac{|y-x|^{2}}{\alpha}+\frac{|z-x|^{2}}{\beta}=\frac{|y-z|^{2}}{\alpha+\beta}+\frac{\alpha+\beta}{\alpha \beta}\left|x-\frac{\beta}{\alpha+\beta} y-\frac{\alpha}{\alpha+\beta} z\right|^{2}$

which is valid for all $x, y$ and $z$ in $\mathbb{R}^{n}$ and for all $\alpha, \beta>0$ and can be verified by a straightforward calculation.

We now apply (3.10), with $\alpha=\frac{u+v+w}{u v}$ and $\beta=\frac{u+v+w}{u w}$, to the first two terms of

$$
\frac{u v|x-y|^{2}+u w|x-z|^{2}+v w|y-z|^{2}}{u+v+w}
$$

which appear in the exponential factor in (2.2). Adding up the third term of (3.11), simplifying, and plugging back into (2.2) we obtain that

$$
\begin{aligned}
\int_{\mathbb{R}^{n}} P_{a}(\tau-x) P_{b}(\tau-y) P_{c}(\tau-z) d \tau & \\
= & \frac{a b c}{\pi^{n+\frac{3}{2}}} \int_{0}^{+\infty} \int_{0}^{+\infty} \int_{0}^{+\infty} \frac{(u v w)^{\frac{n-1}{2}}}{(u+v+w)^{\frac{n}{2}}} \\
& \times \operatorname{Exp}\left[-\left\{a^{2} u+b^{2} v+c^{2} w+\frac{v w}{v+w}|y-z|^{2}\right.\right. \\
& \left.\left.\quad+\frac{u(v+w)}{u+v+w}\left|x-\frac{v}{v+w} y+\frac{w}{v+w} z\right|^{2}\right\}\right] d u d v d w .
\end{aligned}
$$

The change of variables $v=\lambda u$ and $w=\mu u$ transforms the above integral into

$$
\frac{a b c}{\pi^{n+\frac{3}{2}}} \int_{0}^{+\infty} \int_{0}^{+\infty} \frac{(\lambda \mu)^{\frac{n-1}{2}}}{(1+\lambda+\mu)^{\frac{n}{2}}} d \lambda d \mu \int_{0}^{+\infty} u^{n+\frac{1}{2}} e^{-W u} d u
$$

where

$W=a^{2}+b^{2} \lambda+c^{2} \mu+\frac{\lambda \mu}{\lambda+\mu}|y-z|^{2}+\frac{\lambda+\mu}{1+\lambda+\mu}\left|x-\frac{\lambda}{\lambda+\mu} y+\frac{\mu}{\lambda+\mu} z\right|^{2}$.

Expressing the inner integral in (3.12) as a Gamma function we obtain

$$
\frac{a b c \Gamma\left(n+\frac{3}{2}\right)}{\pi^{n+\frac{3}{2}}} \int_{0}^{+\infty} \int_{0}^{+\infty} \frac{(\lambda \mu)^{\frac{n-1}{2}}}{(1+\lambda+\mu)^{\frac{n}{2}} W^{n+\frac{3}{2}}} d \lambda d \mu .
$$


Now let us set $t=\frac{1}{1+\lambda+\mu}$ and $s=\frac{\lambda}{\lambda+\mu}$. This is an invertible transformation of the first quadrant of the $(\lambda, \mu)$ plane onto the square $(0,1) \times(0,1)$ of the $(t, s)$ plane. We have $\lambda=\frac{s(1-t)}{t}$ and $\mu=\frac{(1-t)(1-s)}{t}$, while the Jacobian of the transformation is $\frac{1-t}{t^{3}}$. Collecting and simplifying the various powers of $t,(1-t), s$ and $(1-s)$ that appear we deduce that $\int_{\mathbb{R}^{n}} P_{a}(\tau-x) P_{b}(\tau-y) P_{c}(\tau-z) d \tau$ is in fact equal to the expression in the statement of Lemma 3.1.

Proof of Lemma 3.2: To prove the first inequality, we scale the parameters involved so that $B=1$ and thus $0<a \leq 1$. We need to show that

$$
\begin{aligned}
0 & \leq \int_{0}^{1} \frac{t^{\alpha}(1-t)^{\beta}}{\left\{a^{2} t+(1-t)+t(1-t) D^{2}\right\}^{\gamma-1}}\left(\frac{1}{a}-\frac{1}{a^{2} t+(1-t)+t(1-t) D^{2}}\right) d t \\
& =\frac{1}{a} \int_{0}^{1} \frac{t^{\alpha}(1-t)^{\beta}}{\left\{a^{2} t+(1-t)+t(1-t) D^{2}\right\}^{\gamma}}\left(a^{2} t+1-t-a+t(1-t) D^{2}\right) d t .
\end{aligned}
$$

But $a^{2} t+1-t-a+t(1-t) D^{2} \geq a^{2} t+1-t-a=(1-a)(1-t(1+a))$ and therefore we need to show that

$$
\int_{0}^{1} \frac{t^{\alpha}(1-t)^{\beta}}{\left\{a^{2} t+(1-t)+t(1-t) D^{2}\right\}^{\gamma}}(1-(1+a) t) d t \geq 0 .
$$

As $a^{2} t+(1-t) \leq 1$ and $1-(1+a) t \geq 1-2 t$, it will be sufficient to prove that

$$
\int_{0}^{1} \frac{t^{\alpha}(1-t)^{\beta}}{\left\{1+t(1-t) D^{2}\right\}^{\gamma}}(1-2 t) d t \geq 0 .
$$

We split this integral in the parts from 0 to $\frac{1}{2}$ and from $\frac{1}{2}$ to 1 . Switching variables $t \rightarrow 1-t$ in the second of these integrals we obtain

$$
\int_{0}^{\frac{1}{2}} \frac{(t(1-t))^{\alpha}}{\left\{1+t(1-t) D^{2}\right\}^{\gamma}}\left((1-t)^{\beta-\alpha}-t^{\beta-\alpha}\right)(1-2 t) d t \geq 0
$$

and the first inequality in the lemma is proved. Note that here we have used the hypothesis $\beta>\alpha$. 
To prove the second inequality in Lemma 3.2 we start with the elementary fact

$$
\begin{aligned}
& a^{2} t+(1-t) B^{2}+t(1-t) D^{2}=B^{2}-t\left(B^{2}-a^{2}-D^{2}\right)-t^{2} D^{2} \\
& \geq B^{2}-t\left(B^{2}-a^{2}-D^{2}\right)-t^{2}\left(a^{2}+D^{2}\right)=(1-t)\left[B^{2}+t\left(a^{2}+D^{2}\right)\right]
\end{aligned}
$$

which yields

$$
\left\{a^{2} t+(1-t) B^{2}+t(1-t) D^{2}\right\}^{-\gamma+1} \leq(1-t)^{-\gamma+1}\left\{B^{2}+t\left(a^{2}+D^{2}\right)\right\}^{-\gamma+1} .
$$

Multiplying both sides by $(1-t)^{\beta}$ and taking the integral from 0 to 1 with respect to the weight $t^{\alpha}$ we obtain the second claimed inequality in the lemma. Note that the exponent $\beta-\gamma+1$ can be negative, but $\beta-\gamma+1>-1$ by our assumptions, so that integrability in $t=1$ is guaranteed. Lemma 3.2 is proved.

\section{Proof of Theorem 1.1: the general case}

In this section we outline how to derive the estimate

$$
\left\|P^{*}\left(\chi_{E}, \mu\right)\right\|_{L^{k}\left(\mathbb{R}^{n}\right)}^{k} \leq C^{k}(k !)^{\frac{3}{2}} n^{\frac{k-2}{2}} \kappa(\mu)^{k-1} \mu(E), \quad k=4,5, \ldots
$$

for some absolute constant $C>0$, independent of $n$ and $k$ and for all $\mu$-measurable subsets $E$ of $\mathbb{R}_{+}^{n+1}$.

As a consequence of (4.1) we obtain

$$
\|P f\|_{L^{\frac{k}{k-1}, \infty}\left(\mathbb{R}_{+}^{n+1}, d \mu\right)} \leq C k^{\frac{3}{2}} n^{\frac{1}{2}-\frac{1}{k}} \kappa(\mu)^{\frac{k-1}{k}}\|f\|_{L^{\frac{k}{k-1}}\left(\mathbb{R}^{n}\right)}
$$

for all $k=3,4, \ldots$. These estimates extend (3.1) and (3.2), respectively. Once (4.2) is known, we deduce (1.8) as follows: for a given $p \in(1,2)$ we find a positive integer $k_{0} \geq 4$ such that $k_{0} /\left(k_{0}-1\right)<(p+1) / 2$. We use the Marcinkiewicz interpolation theorem to interpolate between estimate (1.5) (i.e. $p=2$ ) and estimate (4.2) with $p=k_{0} /\left(k_{0}-1\right)$. Using a good value for the interpolation constant (see for instance the value of the constant in $\left[\mathbf{G}\right.$, p. 33]) we obtain (1.8) with $C_{p}=(p-1)^{-\frac{5}{2}}$. We note that the standard proofs of (1.1) yield the constant $c_{p, n}=C(p-1)^{-1} n^{c n}$ (for some absolute $C>0$ and $c>0$ ), which grows much faster in $n$, but blows up at a slightly slower rate when $n$ is fixed and $p \rightarrow 1$. 
In order to work with (4.1) as in the case $k=3$, we need a suitable generalization of Lemma 3.1 for arbitrary $k$. First, we have

$$
\begin{aligned}
\int_{\mathbb{R}^{n}} \prod_{j=1}^{k} P_{a_{j}}\left(\tau-x_{j}\right) d \tau & \\
= & \frac{a_{1} a_{2} \ldots a_{k}}{\pi^{(k-1) \frac{n}{2}+\frac{k}{2}}} \int_{[0, \infty)^{k}} \frac{\left(u_{1} u_{2} \ldots u_{k}\right)^{\frac{n-1}{2}}}{\left(u_{1}+u_{2}+\cdots+u_{k}\right)^{\frac{n}{2}}} \\
& \times \operatorname{Exp}\left\{-\sum_{j=1}^{k} a_{j}^{2} u_{j}-\sum_{\ell=1}^{k-1} \frac{\left(u_{1}+\cdots+u_{\ell}\right) u_{\ell+1}}{u_{1}+\cdots+u_{\ell+1}}\right. \\
& \left.\times\left|x_{\ell+1}-\frac{x_{1} u_{1}+\cdots+x_{\ell} u_{\ell}}{u_{1}+\cdots+u_{\ell}}\right|\right\} d u_{1} \ldots d u_{k}
\end{aligned}
$$

which can be obtained from the $n$-dimensional identity (3.10) working in a recursive fashion as in the proof of Lemma 3.1, until the $k(k-$ 1) $/ 2$ distances appearing in the original formula (2.1) are "squeezed" together into $k-1$ distances. Note that in the case $k=3$ we applied (3.10) just once.

Next, if $\Delta_{k}$ denotes the $(k-1)$-dimensional simplex

$$
\Delta_{k}=\left\{v=\left(v_{1}, \ldots, v_{k}\right): v_{j} \geq 0, \sum_{j=1}^{k} v_{j}=1\right\}
$$

then the change of variables $u=\rho v$ with $v \in \Delta_{k}$ and $\rho>0$ yields

$$
\begin{aligned}
\int_{\mathbb{R}^{n}} & \prod_{j=1}^{k} P_{a_{j}}\left(\tau-x_{j}\right) d \tau \\
= & \frac{a_{1} a_{2} \ldots a_{k} \Gamma\left(\frac{k-1}{2} n+\frac{k}{2}\right)}{\pi^{\frac{k-1}{2} n+\frac{k}{2}}} \int_{\Delta_{k}}\left(v_{1} \ldots v_{k}\right)^{\frac{n-1}{2}} \\
& \times\left(\sum_{j=1}^{k} a_{j}^{2} v_{j}+\sum_{\ell=1}^{k-1} \frac{\left(v_{1}+\cdots+v_{\ell}\right) v_{\ell+1}}{v_{1}+\cdots+v_{\ell+1}}\right. \\
& \left.\quad \times\left|x_{\ell+1}-x_{1} v_{1}+\cdots+x_{\ell} v_{\ell} v_{1}+\cdots+v_{\ell+1}\right|\right)^{-\frac{k-1}{2} n-\frac{k}{2}} d v_{1} \ldots d v_{k}
\end{aligned}
$$


The further change of variables $v_{j}=(1-t) s_{j}, j=1,2, \ldots, k-1$, $v_{k}=t$, with $s=\left(s_{1}, \ldots, s_{k-1}\right) \in \Delta_{k-1}$ and $t \in[0,1]$ gives

$$
\begin{aligned}
\int_{\mathbb{R}^{n}} \prod_{j=1}^{k} P_{a_{j}}\left(\tau-x_{j}\right) d \tau=\frac{a_{1} a_{2} \ldots a_{k} \Gamma\left(\frac{k-1}{2} n+\frac{k}{2}\right)}{\pi^{\frac{k-1}{2} n+\frac{k}{2}}} \\
\quad \times \int_{\Delta_{k-1}} \int_{0}^{1} \frac{(1-t)^{\frac{k-1}{2} n+k-2} t^{\frac{n-1}{2}}\left(s_{1} \ldots s_{k-1}\right)^{\frac{n-1}{2}} d t d s}{\left(a_{k}^{2} t+(1-t) B_{k}^{2}+t(1-t)\left|x_{k}-q_{k}\right|^{2}\right)^{\frac{k-1}{2} n+\frac{k}{2}}}
\end{aligned}
$$

with

$$
\begin{gathered}
q_{k}=x_{1} s_{1}+\cdots+x_{k-1} s_{k-1} \\
B_{k}^{2}=\sum_{j=1}^{k-1} a_{j}^{2} s_{j}+\sum_{\ell=1}^{k-2} \frac{\left(s_{1}+\cdots+s_{\ell}\right) s_{\ell+1}}{s_{1}+\cdots+s_{\ell+1}}\left|x_{\ell+1}-\frac{x_{1} s_{1}+\cdots+x_{\ell} s_{\ell}}{s_{1}+\cdots+s_{\ell+1}}\right|
\end{gathered}
$$

and this is a suitable generalization of Lemma 3.1, with the property that $B_{k}$ is independent of $t, x_{k}, a_{k}$.

We now apply Lemma 3.2 with $\alpha=\frac{n-1}{2}, \beta=\frac{k-1}{2} n+k-2$, $\gamma=\frac{k-1}{2} n+\frac{k}{2}$ and proceed as we did in the previous case $k=3$, assuming $a_{k} \leq a_{k-1} \leq \cdots \leq a_{1}$. We obtain

$$
\begin{gathered}
\int_{\mathbb{R}^{n} \times\left(0, a_{k-1}\right)} a_{k} \int_{0}^{1} \frac{t^{\frac{n-1}{2}}(1-t)^{\frac{k-1}{2}} n+k-2}{\left(a_{k}^{2} t+(1-t) B_{k}^{2}+t(1-t)\left|x_{k}-q_{k}\right|^{2}\right)^{\frac{k-1}{2} n+\frac{k}{2}}} d t d \mu\left(x_{k}, a_{k}\right) \\
\leq \frac{\kappa(\mu) \pi^{n / 2+1}\left(\frac{k-1}{2} n+\frac{k}{2}-1\right) \Gamma\left(\frac{(k-2)(n+1)}{2}\right)}{\left(B_{k}^{2}\right)^{\frac{k-2}{2} n+\frac{k+1}{2}} \Gamma\left(\frac{(k-1) n}{2}+\frac{k}{2}\right)} \\
=\frac{\kappa(\mu) \pi^{n / 2+1} \Gamma\left(\frac{(k-2)(n+1)}{2}\right)}{\left(B_{k}^{2}\right)^{\frac{k-2}{2} n+\frac{k+1}{2}} \Gamma\left(\frac{(k-1)(n+1)}{2}-\frac{1}{2}\right)}
\end{gathered}
$$


Iterating this estimate $k-3$ more times, and doing one last estimate as in $[\mathbf{V}$, equation (13)] we get, for any $k \geq 4$, that

$$
\begin{aligned}
& \frac{1}{k !} \int_{E^{k-1}} \int_{\mathbb{R}^{n}} \prod_{j=1}^{k} P_{a_{j}}\left(\tau-x_{j}\right) d \tau d x_{2} \ldots d x_{k} \\
& \leq \frac{C \kappa(\mu)^{k-1}}{\left(\frac{k-1}{2} n+\frac{k}{2}-1\right)^{-1}} \frac{\Gamma\left(\frac{(k-2)(n+1)}{2}\right)}{\Gamma\left(\frac{(k-2)(n+1)}{2}-\frac{1}{2}\right)} \frac{\Gamma\left(\frac{(k-3)(n+1)}{2}\right)}{\Gamma\left(\frac{(k-3)(n+1)}{2}-\frac{1}{2}\right)} \cdots \\
& \cdots \frac{\Gamma(n+1)}{\Gamma\left(n+\frac{1}{2}\right)} \frac{\Gamma\left(\frac{n+1}{2}\right)}{\Gamma\left(\frac{n}{2}+1\right)}
\end{aligned}
$$

which is of the order of

$$
\kappa(\mu)^{k-1} \sqrt{(k-1) !}(n+1)^{\frac{k-3}{2}+\frac{1}{2}} \sim C^{k} \kappa(\mu)^{k-1} \Gamma(k)^{\frac{1}{2}} n^{\frac{k-2}{2}} .
$$

Since this estimate is uniform with respect to $\left(x_{1}, a_{1}\right)$ we obtain (4.1).

To prove (1.9) we first note that applying the Marcinkiewicz interpolation theorem between (1.5) (i.e. $p=2$ ) and the trivial $p=\infty$ estimate yields

$$
\|P f\|_{L^{p}\left(\mathbb{R}_{+}^{n+1}, d \mu\right)} \leq c^{\prime} p^{\frac{1}{p}}(p-2)^{-\frac{1}{p}} \kappa(\mu)^{\frac{1}{p}}\|f\|_{L^{p}\left(\mathbb{R}^{n}\right)},
$$

for all $p>2$. Finally, using the Riesz-Thorin interpolation theorem, we interpolate between (1.8) with $p=\frac{3}{2}$ and (4.3) with $p=\left(\frac{1}{2}-\frac{1}{\log n}\right)^{-1}$ $(n>10)$ to obtain $(1.9)$.

\section{References}

[A] M. Andersson, "Topics in complex analysis", Universitext, Springer-Verlag, New York, 1997.

[C1] L. CARleson, An interpolation problem for bounded analytic functions, Amer. J. Math. 80 (1958), 921-930.

[C2] L. Carleson, Interpolations by bounded analytic functions and the corona problem, Ann. of Math. (2) 76 (1962), 547-559.

[G] L. Grafakos, "Classical and Modern Fourier Analysis", Pearson Education, Upper Saddle River, New Jersey, 2004.

[H] L. HöRmANDER, $L^{p}$ estimates for (pluri-) subharmonic functions, Math. Scand. 20 (1967), 65-78.

[N] N. K. NikOL'SKIĬ, "Treatise on the shift operator", Spectral function theory, With an appendix by S. V. Hruščev and V. V. Peller, Translated from the Russian by Jaak Peetre, Grundlehren der 
Mathematischen Wissenschaften 273, Springer-Verlag, Berlin, 1986.

[S] E. M. STEIN, "Harmonic analysis: real-variable methods, orthogonality, and oscillatory integrals", With the assistance of Timothy S. Murphy, Princeton Mathematical Series 43, Monographs in Harmonic Analysis III, Princeton University Press, Princeton, NJ, 1993.

[SW] E. M. SteIn AND G. WeISS, "Introduction to Fourier analysis on Euclidean spaces", Princeton Mathematical Series 32, Princeton University Press, Princeton, N.J., 1971.

[V] I. E. Verbitsky, A dimension-free Carleson measure inequality, in: "Complex analysis, operators, and related topics", Oper. Theory Adv. Appl. 113, Birkhäuser, Basel, 2000, pp. 393-398.

Loukas Grafakos:

Department of Mathematics

University of Missouri

Columbia, Missouri 65211

U.S.A.

E-mail address: loukas@math.missouri.edu

Enrico Laeng:

Dipartimento di Matematica

Politecnico di Milano

Piazza L. da Vinci 32

20133 Milano

Italy

E-mail address: enrlae@mate.polimi.it

Carlo Morpurgo:

Department of Mathematics

University of Missouri

Columbia, Missouri 65211

U.S.A.

E-mail address: morpurgo@math.missouri.edu

Primera versió rebuda el 25 d'abril de 2006,

darrera versió rebuda el 3 d'octubre de 2006. 